\title{
Microcystic Pancreatic Ductal Adenocarcinoma: Myth or Reality?
}

We present the case of a 48-year-old male with pain in the upper abdomen and jaundice for 2 months. Minimally deranged liver function tests were seen in the form of mild elevation of total serum bilirubin - $1.16 \mathrm{mg} / \mathrm{dL}(N$ : $0.3-1.2 \mathrm{mg} / \mathrm{dL})$ and raised alkaline phosphatase levels - 307 IU/L ( $N$ : 20-140 IU/L) with normal serum glutamic pyruvic transaminase/serum glutamic-oxaloacetic transaminase 35/20 IU/L. Hewas evaluated with contrast-enhanced computed tomography (CECT) and magnetic resonance cholangiopancreatography (MRCP). CECT revealed multicystic mass in the head of the pancreas [Figure 1a]. In view of the raised bilirubin/ cholangitis, endoscopic retrograde cholangiopancreatography with stenting was done. MRCP confirmed the presence of a honeycomb cystic lesion in the head of the pancreas having communication with the main pancreatic duct with mild dilatation of the side-duct branches [Figure 1b]. A provisional diagnosis of branch-duct intraductal papillary mucinous neoplasm (IPMN) was made considering the morphology of the lesion. After taking due consent and fitness, he underwent open classic pancreatoduodenectomy. Postoperative histopathological examination revealed a highly infiltrative tumor composed of cysts of variable sizes separated by paucicellular desmoplastic stroma. The epithelial cells lining these cysts were cuboidal to low columnar and showed moderate nuclear pleomorphism and atypia. Frequent mitotic figures, including atypical ones, were seen (10-12/10 high-power field). At places, the nuclear stratification of the lining epithelial cells was noted. The lining epithelial cells of cysts showed positive staining for p53 and membranous staining for carcinoembryonic antigen, making the diagnosis of microcystic variant of pancreatic adenocarcinoma [Figure 2a-d] over IPMN. Pancreatic ductal adenocarcinoma (PDAC) with large-duct type cysts is one of the morphologic variations of PDACs, which is characterized by more dilated malignant ducts forming a microcystic feature. The size of cysts is small, usually $0.5-0.7 \mathrm{~cm}$ in

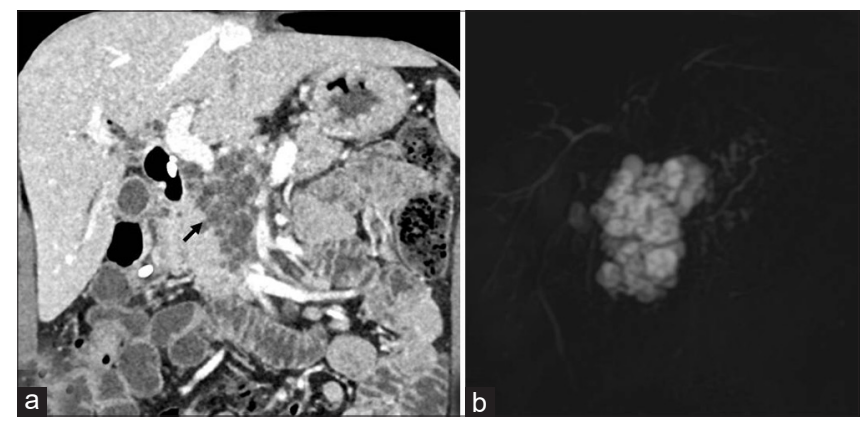

Figure 1: (a) Contrast-enhanced computed tomography showing microcystic mass (arrow) in the pancreatic head. (b) Magnetic resonance cholangiopancreatography confirming a honeycomb cystic lesion in the head of the pancreas having communication with the main pancreatic duct with mild dilatation of the side-duct branches diameter, occasionally exceeding $1 \mathrm{~cm}$ and can mimic IPMN or microcystic serous cystadenoma on imaging.

PDAC typically presents as an irregular solid tumor with a scirrhous character resulting from a prominent desmoplastic reaction. The incidence of PDAC with cystic changes ranges from $<1 \%$ to $8 \%$. PDACs with cystic change are uncommon and may occur by means of the four following mechanisms - (i) obstruction of the pancreatic duct by the tumor resulting in the formation of retention cysts localized outside the tumor periphery, (ii) coexisting acute pancreatitis resulting in pseudocyst formation localized outside the tumor, (iii) microcystic appearance caused by marked ectasia of the invasive gland and referred to as the "large-duct type," and (iv) necrosis within the tumor. The large-duct type ectasia within the tumor was seen in the present case.

\section{Declaration of patient consent}

The authors certify that they have obtained all appropriate patient consent forms. In the form, the patient has given his consent for his images and other clinical information to be reported in the journal. The patient understands that his name and initial will not be published, and due efforts will be made to conceal his identity, but anonymity cannot be guaranteed.

Financial support and sponsorship

Nil.

\section{Conflicts of interest}

There are no conflicts of interest.

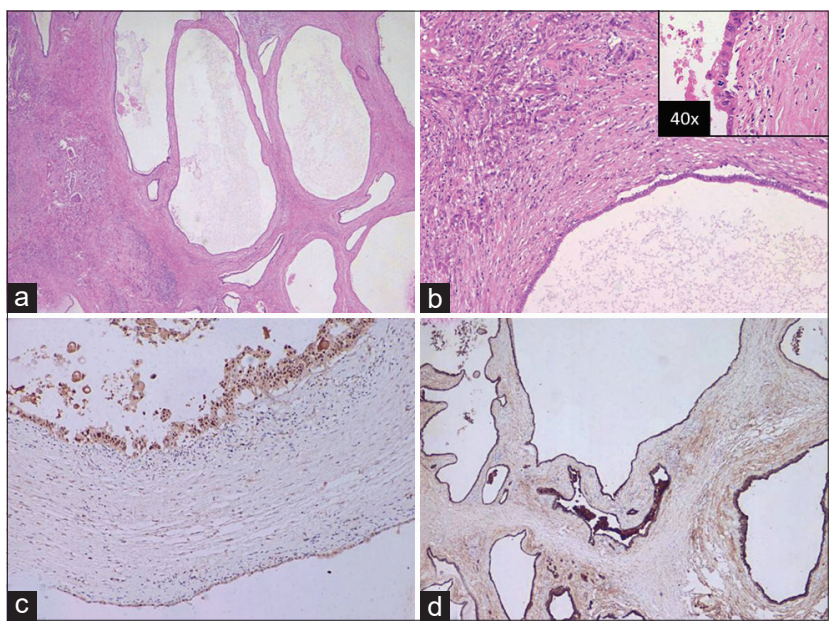

Figure 2: (a) Low-power microphotograph showing cyst of variable size with epithelial lining, separated by paucicellular desmoplastic stroma ( $H$ and $E, \times 20)$. (b) Further magnification shows that the cyst is lined by cuboidal epithelial cells. The small irregular angulated glands are also noted $(H$ and $E \times 100)$. Inset high-power image $(\times 40)$ shows moderate nuclear atypia of lining epithelial cells and occasional atypical mitotic figures. (c and d) The lining epithelial cells of cysts show nuclear expression of p53 $(\times 100)$ and membranous staining for carcinoembryonic antigen $(\times 40)$ 


\section{Binit Sureka ${ }^{1}$, Vikarn Vishwajeet ${ }^{2}$, Poonam Elhence ${ }^{2}$, Vaibhav Varshney ${ }^{3}$, Taruna Yadav ${ }^{1}$}

${ }^{\prime}$ Department of Diagnostic and Interventional Radiology, All India Institute of Medical Sciences, Jodhpur, Rajasthan, India, ${ }^{2}$ Department of Pathology and Lab Medicine, All India Institute of Medical Sciences, Jodhpur, Rajasthan, India, ${ }^{3}$ Department of Surgical Gastroenterology, All India Institute of Medical Sciences, Jodhpur, Rajasthan, India

Address for correspondence: Dr. Binit Sureka,

Department of Diagnostic and Interventional Radiology, All India Institute of Medical Sciences, Jodhpur - 342 005, Rajasthan, India. E-mail: binitsurekapgi@gmail.com

Submitted: 28-Jun-2020

Revised: 23-Jul-2020

Accepted: 19-Aug-2020

Published: 29-Oct-2020
This is an open access journal, and articles are distributed under the terms of the Creative Commons Attribution-NonCommercial-ShareAlike 4.0 License, which allows others to remix, tweak, and build upon the work non-commercially, as long as appropriate credit is given and the new creations are licensed under the identical terms.

\begin{tabular}{|l|l|}
\hline \multicolumn{2}{|c|}{ Access this article online } \\
\hline Quick Response Code: & Website: \\
& www.ijmpo.org \\
\cline { 2 - 2 } & Dol: \\
\hline
\end{tabular}

How to cite this article: Sureka B, Vishwajeet V, Elhence P, Varshney V, Yadav T. Microcystic pancreatic ductal adenocarcinoma: Myth or reality? Indian J Med Paediatr Oncol 2020;41:731-2. 